\title{
Exteriorización de una prótesis PTFE en una paciente desmotivada
}

\author{
Esperança Anguera Guinovart - Eulàlia Oriol Colominas
}

Enfermeras

Unidad de Diálisis. Hospital Universitario Joan XXIII de Tarragona.

\section{Introducción}

Uno de los principales retos es conseguir un acceso vascular idóneo para los pacientes que precisan hemodiálisis. La fístula arteriovenosa (FAV) autóloga es el acceso vascular permanente de elección. Cuando esta opción no es posible, se debería optar por una prótesis vascular de politetrafluoroetileno (PTFE) y en tercer lugar por un catéter permanente tunelizado $(\mathrm{CPT})^{1-2}$.

La inclusión en diálisis de pacientes de edad avanzada, con mayor incidencia de diabetes, de enfermedad cardiovascular y la hemodiálisis de larga evolución ha hecho que el número de prótesis y de catéteres, aumente considerablemene ${ }^{3}$.

Las prótesis, se unen por un extremo a la arteria y por el otro a la vena mediante un material sintético, en la actualidad, politetrafluoroetileno (PTFE) ${ }^{1,2}$. En España, el porcentaje de pacientes que usan una prótesis para hemodiálisis (PPHD) oscila entre el 5\% y el $25 \%$.

Este tipo de acceso proporciona altos flujos de sangre ${ }^{3}$, es fácil de canalizar, y no ofrece resistencia de retorno,

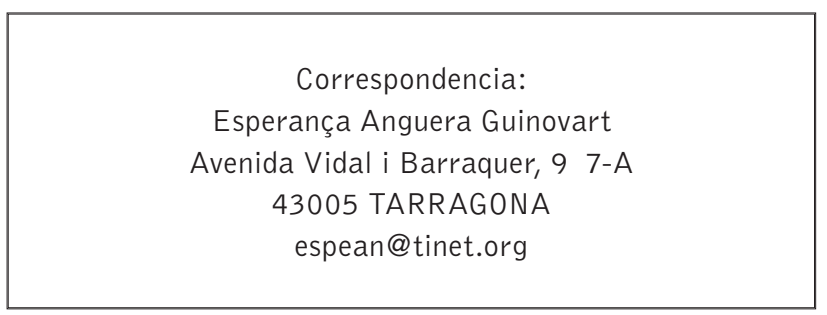

pero también es cierto que presenta más complicaciones que las FAVI autólogas y además éstas aparecen más tempranamente ${ }^{6}$.

La complicación más frecuente en los PTFE es la trombosis $^{6}$. Menos frecuente, es el edema de extremidad por hipertensión venosa central que suele asociarse a estenosis o trombosis de las venas centrales, generalmente debido a la utilización de la vía central como acceso vascular ${ }^{7}$.

Las complicaciones infecciosas son también de relevante importancia. Destacamos la posibilidad de infección del PTFE trombosado, en estos casos, la infección suele desarrollarse de forma subclínica por lo que es difícil un diagnóstico precoz $z^{8}$.

Otra de las complicaciones habituales en las PPHD son los pseudoaneurismas o falsos aneurismas, manifestados como una masa pulsátil generalmente no dolorosa ${ }^{3}$. Relativamente frecuentes son las hemorragias, o la rotura del acceso vascular normalmente secundaria a un traumatismo y sobre todo asociada a pseudoaneurismas o infecciones del acceso vascular. No menos importante es la isquemia por síndrome de robo favorecida por la existencia del acceso ${ }^{7}$.

Es importante realizar una inspección sistemática del acceso para detectar y tratar precozmente complicaciones $y / 0$ lesiones potencialmente responsables de una disfunción o del buen funcionamiento del AV. En la mayoría de unidades de HD se han desarrollado protocolos de monitorización del acceso vascular?. Gracias a ello, podemos realizar un meticuloso segui- 
miento y un adecuado tratamiento de sus múltiples complicaciones, alargando así la vida de las prótesis PTFE para diálisis.

A continuación, presentamos el caso clínico de una exteriorización espontánea de una prótesis PTFE para diálisis, complicación insólita, ya que revisando bibliografía, no hemos encontrado ningún caso similar.

\section{Caso clínico}

\section{Antecedentes}

Paciente de 56 años, laboralmente activa, madre con HTA, padre con antecedentes de IAM. Paciente sin alergias medicamentosas, enolismo crónico, ex-fumadora, HTA severa de 8 años de evolución en tratamiento farmacológico, DM tipo II secundaria a pancreatitis crónica, en tratamiento con insulina, hepatitis en la infancia, hemobilia secundaria a biopsia hepática. Biopsia de riñón por punción: nefritis túbulo intersticial crónica con nefroangiosclerosis. IRC en hemodiálisis desde 1998. Hiperparatiroidismo secundario severo, declinando de manera reiterada la paratireidectomia.

En junio de 1998 la paciente inicia diálisis. En agosto del mismo año se le practica una FAVI radial I, después de 4 años se procede a la ligadura de dicho acceso por hemorragia severa. Aquí empieza un extenso historial de intervenciones para obtener un acceso vascular: FAVI en codo $D$, prótesis arteriovenosas PTFE en ambas piernas, catéteres en ambas venas yugulares con dificultad de canalización, catéteres en venas subclavias y femorales. Por imposibilidad de obtener acceso vascular, en julio de 2005 inicia diálisis peritoneal.

\section{Exteriorización de la prótesis PTFE}

En marzo de 2006 la paciente acude a nuestro servicio desde urgencias por presentar exteriorización espontánea de la prótesis PTFE en extremidad inferior izquierda, de pocas horas de evolución, con signos de infección de la herida, zona inflamada y enrojecida, sin fiebre y sin dolor en la zona afectada. La prótesis queda al descubierto, prácticamente colgando sobre el muslo, la paciente no ha detectado la apertura de la herida ni el comienzo de la exteriorización. (veáse foto I y II).

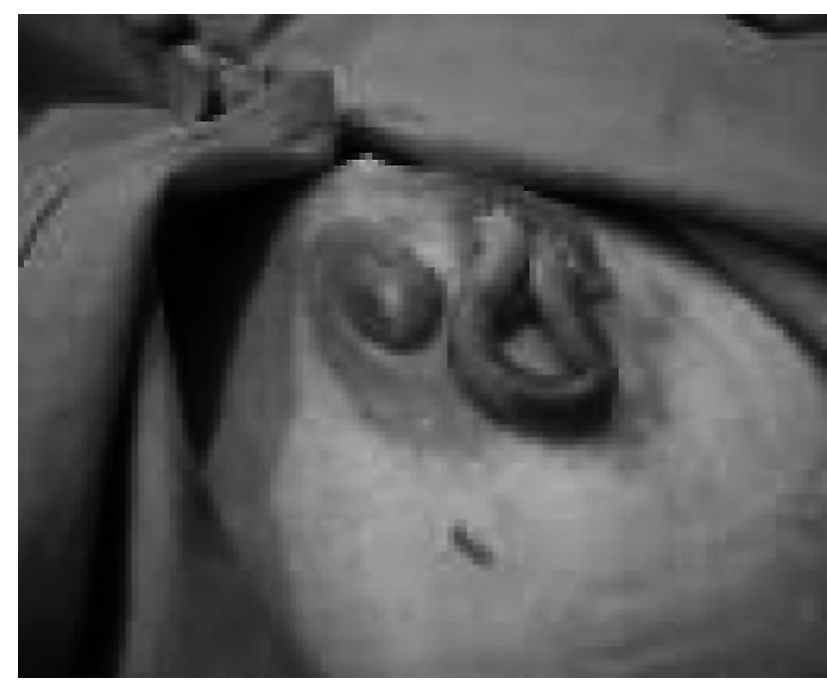

Foto I. Prótesis PTFE al descubierto en muslo

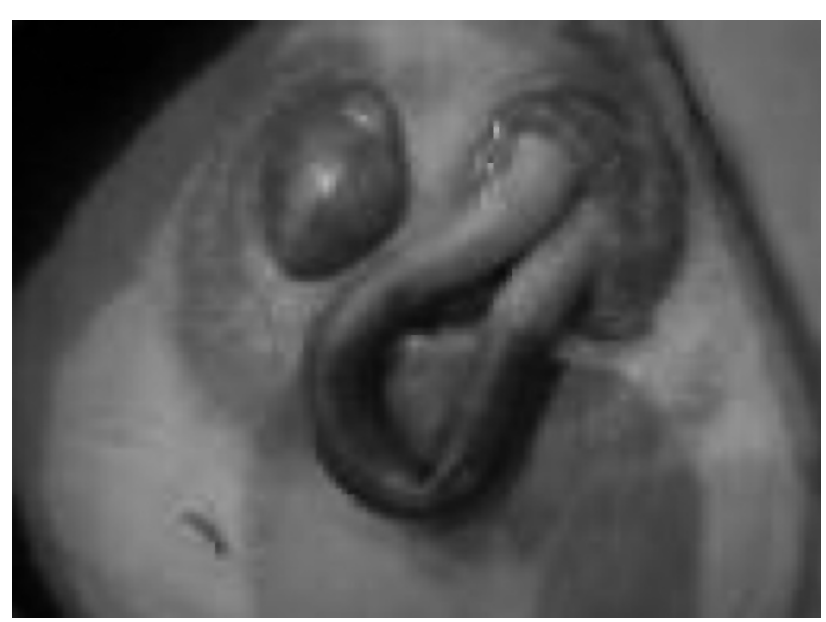

Foto II. Prótesis PTFE exteriorizada en muslo

Esta prótesis arteriovenosa PTFE llevaba un año implantada en extremidad inferior I (se realizó una tunelización subcutánea con anastomosis termino-lateral venosa y termino-lateral arterial), pero nunca llegó a usarse ya que se trombosó un mes después de ser implantada. La paciente refiere desánimo e indiferencia, mostrándose poco expresiva.

\section{Exploración física}

TA 131/75 mmHg. FC 54 px'. T $^{\mathrm{a}} 36^{\circ} \mathrm{C}$. Estado general conservado. Normocoloreada. Heridas en muslo izquierdo con signos inflamatorios y con exteriorización 
de prótesis vascular. Signos de hiperhidratación con importantes edemas con fóvea en ambas extremidades hasta rodilla. Eupneica en reposo. AC: tonos rítmicos, no auscultación de soplos. AR: hipofonesis en base pulmonar izquierda. Abdomen blando y depresible, no doloroso a la palpación.

\section{Intervención enfermería}

Se irriga toda la zona afectada con suero fisiológico y después con povidona yodada, se cubre con una talla estéril y se traslada a quirófano donde la paciente es intervenida por el servicio de Cirugía Vascular (retirada de PTFE de EII con interposición de arteria femoral superficial termino-terminal, toma de cultivos y retirada de granulomas). Se inicia tratamiento empírico con Ceftriaxona. El cultivo de la herida quirúrgica da positivo por S. Epidermidis, sensible al tratamiento antibiótico.

La herida quirúrgica se cura cada 24 horas con suero fisiológico y povidona yodada.

Se realizan sesiones de diálisis peritoneal con aumento en la concentración de glucosa del líquido de diálisis, consiguiendo así disminución de los edemas y del estado de hipervolemia.

Se retiran la mitad de grapas a los diez días y el resto al día siguiente. La herida quirúrgica ha cerrado sin complicaciones y han desaparecido los signos de inflamación. Es dada de alta.

\section{Desenlace}

En septiembre de 2006 (seis meses después de la retirada de la prótesis exteriorizada) ingresa por peritonitis. Los familiares traen a la paciente al hospital en mal estado general y con disminución de la conciencia. La paciente insinúa que lleva días sin realizar pases peritoneales. Refiere cansancio y desánimo y se muestra indiferente. De esta situación deriva el incumplimiento del tratamiento peritoneal, el descuido del estado nutricional y el abandono de cualquier aspecto relacionado con el propio cuidado. Se coloca catéter en vena femoral para hemodiálisis que, dada la inestabilidad hemodinámica de la paciente, se realiza a bajo flujo durante 6 horas. Presenta úlceras en los dos glúteos, coxis y una úlcera en ambas extremidades inferiores. Las úlceras se curan diaria- mente con pomada desbridante, apósitos hidrocoloides y povidona yodada, según el tipo de úlceras. Después de ocho días de ingreso es derivada a un centro socio sanitario, anímicamente parece triste.

Seis días después reingresa, con caquexia severa, por síndrome febril atribuido a una sobreinfección de las úlceras cutáneas. Las úlceras son sugestivas de calcifilaxis por hiperparatiroidismo secundario severo. PTH superior a 1900 pg./ml que tras tratamiento baja a 300 pg./ml. Se realiza biopsia cutánea. Los cultivos de la úlcera confirman la infección y se instaura tratamiento antibiótico, además de la Eritropoyetina también precisó tres concentrados de hematíes. Se hicieron curas cada 24 horas de las heridas con desbridamiento de esfacelos y de zonas necróticas.

Por imposibilidad de obtener acceso vascular para hemodiálisis se suspendió el programa de hemodiálisis y se prosiguió con diálisis peritoneal con administración intraperitoneal de Nutrineal y suplementos orales de aminoácidos.

A pesar de todo ello las úlceras fueron extendiéndose por ambas EEII y en brazos. La paciente fue desarrollando una caquexia progresiva y murió 4 días después de su reingreso.

\section{Discusión y conclusión}

Este es un caso clínico que describe una complicación de prótesis PTFE infrecuente, en una paciente que parece haber perdido la ilusión de vivir. En nuestro servicio, Ilamó la atención por insólito y espectacular. Ante tal hecho, nuestra pregunta es "cómo y porqué ocurrió" y tras un espacio de investigación y reflexión, queremos en primer lugar plantearnos la importancia del cuidado y la revisión del acceso vascular para prevenir y detectar posibles complicaciones. En nuestras unidades, tenemos protocolos para el cuidado del acceso vascular funcionante, pero invitamos a la reflexión sobre la necesidad de revisar todos aquellos accesos vasculares del paciente no funcionantes, trombosados o que están en desuso y crear protocolos para su valoración.

Por otro lado sabiendo que es de vital importancia la implicación y participación del paciente en su auto cuidado, también concluimos que el estado de desánimo y de abandono pudo comportar, por un lado no detectar 
signos de inflamación o infección en la zona de exteriorización de $\mathrm{PTFE}$, así como también el abandono del tratamiento, con la consiguiente hiperhidratación y aparición de importantes edemas, hecho que ha podido influir en la exteriorización de la prótesis PTFE, también el abandono de la medicación y tratamiento contribuyendo a la aparición de calcifilaxis, en el estado de desnutrición y en el deterioro general. Por tanto, a parte la formación y del aprendizaje de los aspectos técnicos, queremos destacar la monitorización de la motivación y del estado de ánimo.

\section{Agradecimientos}

Nuestro agradecimiento a la paciente por lo que hemos aprendido de su comportamiento y de sus silencios. Nuestro especial agradecimiento a la Dra. M del Carme García Ruiz por su gran ayuda durante la realización del caso clínico.

\section{Bibliografía}

1. Guía de Acceso Vascular en Hemodiálisis. Sociedad Española de Nefrología. Noviembre 2004.

2. National Kidney Foundation 2006 updates Clinical practice Guidelines and recommendations KDOQI.

3. Bohórquez-Sierra JC, Doiz-Artázcoz E, ArribasAguilar F, Bohórquez-Sierra C. Accesos Vasculares para hemodiálisis. Complicaciones: aneurismas verdaderos y falsos, hemorragias y roturas del acceso vascular. Angiología 2005; 57 (2): 117-118.
4. Rodríguez Hernández JA, López Pedret J y Piera L. El acceso vascular en España: análisis de su distribución, morbilidad y sistemas de monitorización. Nefrología. 2001; 21(1): 45-51.

5. Lerma-R R, Callejas-P JM. Accesos vasculares para hemodiálisis: equipos multidisciplinarios. Angiología 2005; 57 (I2): 169-176.

6. Blanes Mompó JI, Martínez Rodrigo J, Górriz Teruel JL, Crespo Moreno I, Gómez Palonés F, Martínez Meléndez $S$, et tal. Tratamiento de la trombosis de los injertos de PTFEE para hemodiálisis mediante trombectomía percutánea. Estudio prospectivo. Angiología 2001; 53: 393-403.

7. Sáez-Martín L, Riera-Del Moral L, Gutiérrez-Nistal M, Estefanov E, Riera de Cubas Accesos vasculares para hemodiálisis. Otras complicaciones: isquemia distal, hipertensión venosa distal y trombosis de vasos centrales. Angiólogía 2005; 57 (2): 137-S143.

8. Aparicio-Martínez C, González-García A, del RíoPrego A. Accesos vasculares para hemodiálisis. Complicaciones: infecciones del acceso vascular (autólogo o protésico) Angiología 2005; 57 (2): 129-35.

9. Galera-Fernández A, Martínez-de Merlo MT, Ochando-García A. Accesos vasculares para hemodiálisis: cuidados de enfermería. Angiología 2005; 57 (2): 159-68. 